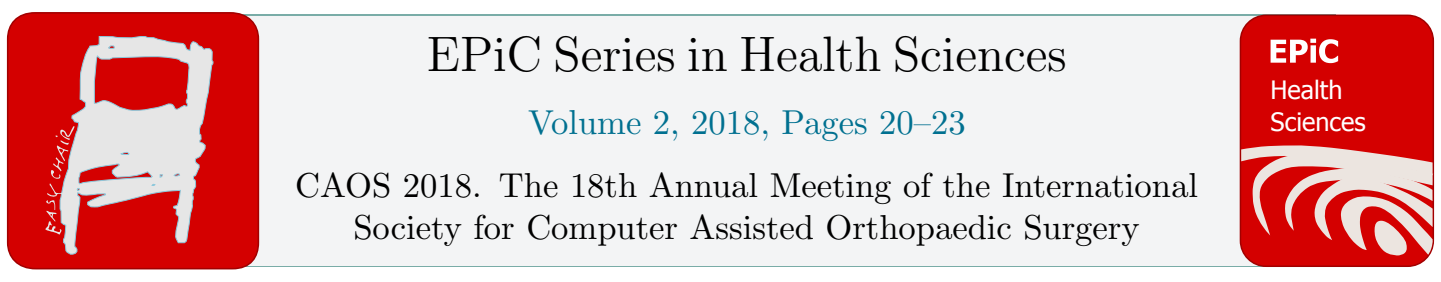

\title{
Coronal Knee Laxity Measured During Computer-Assisted Total Knee Arthroplasty
}

\author{
Yifei Dai ${ }^{1 *}$, Guillaume Bras², David Liu ${ }^{3 *}$ \\ ${ }^{1 *}$ Exactech Inc, Gainesville, FL 32653, USA \\ ${ }^{2}$ Blue Ortho, Gieres, FR \\ ${ }^{3}$ The Gold Coast Centre for Bone and Joint Surgery, Tugun, Queensland, AU \\ yifei.dai@exac.com, dliu01@bigpond.com
}

\begin{abstract}
This observational study on fifty-six patients presents the coronal knee angular laxity measured during computer-assisted total knee arthroplasty. Varus/valgus knee laxity was assessed at extension, mid-flexion, and high flexion, and compared between arthritic knees and knees following trial TKA implantation. The data represented soft tissue laxity under surgical reality and may be used to further understand the clinical implications of intraoperative soft-tissue management. The findings highlight the potential for CAOS to accurately manage soft tissue balance in addition to bony cuts and overall alignment.
\end{abstract}

\section{Introduction}

Appropriate management of the soft tissue envelope intraoperatively is critical to the success of total knee arthroplasty (TKA). Knee laxity has been studied in vitro and in vivo using calibrated force and special fixture devices [1-4]. However, tests under these conditions often represent healthy rather than arthritic knees and are not suitable to be applied intraoperatively. The results reported by the studies therefore may not accurately reflect knee laxity observed in the operating room.

Recently, several computer-assisted orthopaedic surgery (CAOS) systems provide tools for measuring ligamentous balance intraoperatively. While surgeons are using intraoperatively measured soft tissue envelope laxity to inform their surgical decisions, there remains little consensus on what constitutes the ideal varus valgus laxity.

The purpose of this observational study was to quantify coronal laxity of the knee before and after TKA implantation, measured intraoperatively by a single surgeon using a contemporary CAOS system. 


\section{Materials and Methods}

Technical records of cruciate-retaining (CR) TKA cases performed by the surgeon author (DL) using a contemporary CAOS system (ExactechGPS ${ }^{\circledR}$, Blue-Ortho, Gieres, FR) were reviewed. All cases performed since the release of a Kinematic Test Module in the CAOS system were included in this study. All cases were performed using a modified gap balancing technique, with measured resection of the distal femur and proximal tibia, followed by gap balancing of the flexion gap using a tensioner device. The surgeon aimed for equal flexion and extension gaps, and symmetrical mediolateral gaps.

Each case record contained results from manual varus/valgus stress tests across the range of motion (ROM) with patella reduced. The stress tests were performed under two knee conditions: 1) arthritic knee: coronal laxity was tested just after medial parapatellar exposure and attachment of the CAOS trackers; and 2) TKA knee: coronal laxity was tested with trial implantation of the prosthesis.

Coronal laxity at flexion angles of $0^{\circ}, 40^{\circ}$, and $90^{\circ}$ was compared (paired-t test) between test conditions. The specific laxity measures investigated were: maximum varus laxity angle, maximum valgus laxity angle, angular size of the laxity envelope, and asymmetry of the laxity envelope at full extension (midpoint of the varus/valgus laxity at $0^{\circ}$ flexion). Statistical significance was defined as $\mathrm{p}<$ 0.05 .

\section{Results}

Fifty-six cases were reviewed from the surgical record database (Fig. 1). The arthritic knees exhibited significantly more maximum varus and valgus laxity angles during mid-flexion $\left(40^{\circ}\right)$ compared to $0^{\circ}$ and $90^{\circ}$ of flexion (p values $\leq 0.004$ ) (Fig. 2A). Similarly, significant larger coronal laxity envelope was observed for the arthritic knees during mid-flexion than those of $0^{\circ}$ and $90^{\circ}$ of flexion ( $\mathrm{p}$ values $=0.000$ ) (Fig. 2B). At full flexion, the coronal laxity of the arthritic knees was asymmetric, with the mid-point of the envelope deviated on average $3.2^{\circ}$ from the neutral center.

Clear differences in maximum varus and valgus laxity were observed between arthritic and trial TKA implanted knees across the flexion range (Fig. 2). After trial insertion, an elevated laxity during mid flexion was no longer observed. The maximum varus and valgus laxity angles were generally consistent across the flexion range, except for a slightly more maximum varus laxity $\left(<2^{\circ}\right)$ in flexion $\left(40^{\circ}\right.$ and $\left.90^{\circ}\right)$ compared to extension $\left(0^{\circ}\right)$. With TKA trial insertion, both the size and asymmetry of varus/valgus laxity envelope significantly decreased from arthritic knee. After trial implantation at full extension $\left(0^{\circ}\right.$ flexion), all the knees had maximum varus laxity angle equal or less than $3^{\circ}$, while all maximum valgus laxity were within $5^{\circ}$, with the maximum valgus laxity angle slightly higher than maximum varus laxity angle (on average $\sim 1.5^{\circ}$ ).
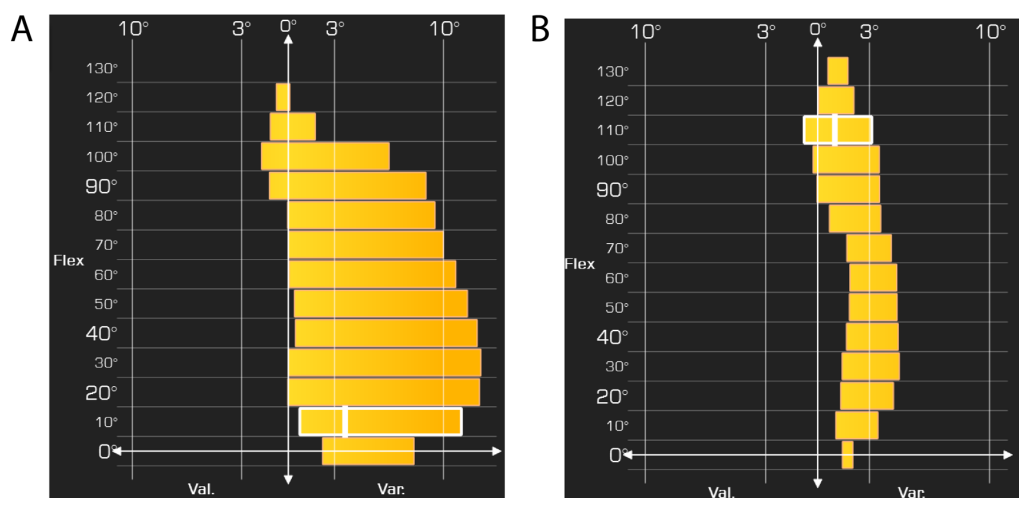
Figure 1. A representative surgical record demonstrating intraoperative varus/valgus laxity test results for the conditions of $\mathrm{A}$ ) arthritic knee and $\mathrm{B}$ ) post TKA implantation.

\begin{tabular}{lccc}
\hline & & Flexion (") & \\
& 0 & 40 & 90 \\
Max Varus Laxity (7) & & & \\
Arthritic Knee & $2.9 \pm 4.6$ & $5.6 \pm 5.5$ & $4.5 \pm 5.8$ \\
TKA & $0.0 \pm 1.4$ & $1.6 \pm 3.6$ & $1.8 \pm 4.8$ \\
P & 0.000 & 0.000 & 0.000 \\
Max Valgus Laxity (?) & & & \\
Arthritic Knee & $-2.2 \pm 4.5$ & $-3.2 \pm 6.0$ & $-2.1 \pm 5.7$ \\
TKA & $-1.4 \pm 1.6$ & $-1.5 \pm 3.6$ & $-1.3 \pm 4.7$ \\
P & 0.064 & 0.007 & 0.033 \\
Laxity Envelope (") & & & \\
Arthritic Knee & $5.0 \pm 2.9$ & $8.8 \pm 3.2$ & $6.6 \pm 3.3$ \\
TKA & $1.4 \pm 1.0$ & $3.2 \pm 2.5$ & $3.4 \pm 2.6$ \\
P & 0.000 & 0.000 & 0.000 \\
\hline
\end{tabular}

A

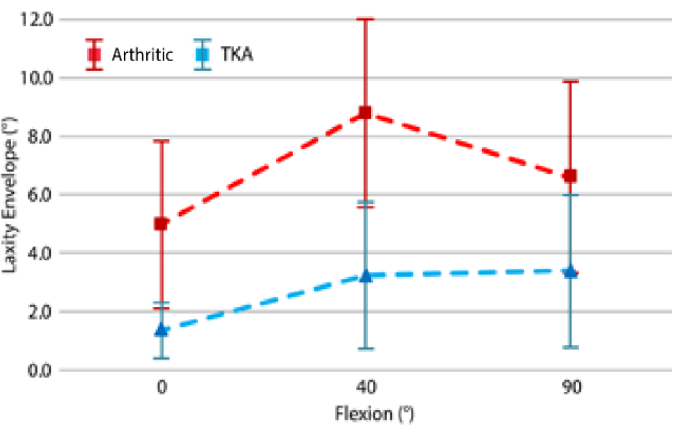

B

Figure 2. A) Summary of measured coronal laxity. B) Comparison of the size of coronal laxity envelope between different flexion angles and the two test conditions.

\section{Discussion}

This study provides insight into the clinically observed passive laxity tested intraoperatively in arthritic knees and knees after trial TKA implantation. The elevated mid-flexion coronal laxity may indicate reduced rotational support provided by passive joint structures in the arthritic knees. TKA surgery significantly reduced varus/valgus laxity across the flexion range and removed the pattern of elevated mid-flexion laxity, reflecting stabilization of the knee joint by the surgical intervention. The implanted knees in this study exhibited the same trend as previous reported data on physiological normal knees, which showed a consistent increase in varus/valgus laxity envelope with increased knee flexion from $0^{\circ}$ to $90^{\circ}$ flexion [6]. In addition, the implanted knees aligned with previous reports that valgus laxity was higher than varus laxity in the normal knee $[7,8]$.

Limitations of the study include a small series from a single surgeon only. The post implantation laxities may be dependent on the load of tensioning device the surgeon selected for balancing, and the measurement based on trial implants may differ following cementation. Further analysis may be carried out to investigate inter-surgeon differences with increased sample size.

In addition to offering the proven benefit on accurate bony resection [5], some CAOS systems have developed tools for the measurement of passive knee kinematics and soft tissue laxity intraoperatively. Studying intraoperatively measured knee laxity by the CAOS system may improve the understanding of ligament balancing and enhance knowledge of what constitutes appropriate soft tissue balance. Once this information is known, the potential to improve CAOS for intraoperative guidance will be enhanced, incorporating bone cuts, overall alignment and ligament laxity. 


\section{References}

[1] Markolf KL, Mensch JS, Amstutz HC. Stiffness and laxity of the knee - the contributions of the supporting structures. A quantitative in vitro study. J Bone Joint Surg Am 1976; 58:583-94.

[2] Van Damme G, Defoort K, Ducoulombier Y, et al. What should the surgeon aim for when performing computer-assisted total knee arthroplasty? J Bone Joint Surg Am 2005;87 (Suppl. 2) :52-8

[3] Heesterbeek PJC, Verdonschot N, Wymenga AB. In vivo knee laxity in flexion and extension: a radiographic study in 30 older healthy subjects. Knee 2008;15:45-9.

[4] TokuharaY, Kadoya Y, Nakagawa S, et al. The flexion gap in normal knees. An MRI study. J Bone Joint Surg Br 2004;86:1133-6.

[5] Sparmann M, Wolke B, Czupalla H, et al. Positioning of total knee arthroplasty with and without navigation support: a prospective, randomised study. J Bone Joint Surg Br 2003;85(6):830-5.

[6] Ghosh KM, Blain AP, Longstaff L, Rushton S, Amis AA, Deehan DJ. Can we define envelope of laxity during navigated knee arthroplasty? Knee Surg Sports Traumatol Arthrosc. 2014;22(8):1736-43.

[7] Okazaki K, Miura H, Matsuda S, et al. Asymmetry of mediolateral laxity of the normal knee. J Orthop Sci 2006;11:264-6.

[8] Yoo JC, Ahn JH, Sung KS, et al. Measurement and comparison of the difference in normal medial and lateral knee joint opening. Knee Surg Sports Traumatol Arthrosc 2006;14:1238-44. 